\title{
Pseudospectral Model Predictive Control for Exo-atmospheric Guidance
}

\author{
Tawfiqur Rahman*, Hao Zhou**, Liang Yang*** and Wanchun Chen**** \\ School of Astronautics, Beihang University, Beijing 100191, China
}

\begin{abstract}
This paper suggests applying pseudospectral model predictive method for exo-atmospheric guidance. The method is a fusion of pseudospectral law and model predictive control, in which a two point boundary value problem is formulated using model predictive approach and solved by applying pseudospectral law. In this work, the method is applied to exo-atmospheric guidance with specific target requirement. The existing exo-atmospheric guidance methods suffice general requirements for guidance, but cannot ensure specific target constraints; whereas, the presented method is able to do so. The proposed guidance law is assessed through simulation of perturbed cases, and the tests suggest that the method is able to operate semiautonomously under control and thrust vector perturbations.
\end{abstract}

Key words: Exo-atmospheric Guidance, Pseudospectral Model Predictive Control, Gauss Pseudospectral Method, Hypersonic Vehicle Guidance, Model Predictive Control

\section{Introduction}

Exo-atmospheric guidance refers to guidance beyond the atmosphere with negligible aerodynamic effects. In vacuum, a vehicle encounters forces from two sources only: its own thrust and gravitation of the planet. Due to the relative simplicity, closed loop optimal or near-optimal solution can be obtained for vacuum guidance. This has been used as an advantage in guidance system of the existing exoatmospheric vehicles. For the past and present missions, vacuum guidance methods have been sufficient. For example, in an orbital injection mission, only the desired orbit and velocity is to be met; for CRV (Crew Return Vehicle), CLV (Crew Launch Vehicle), or CaLV (Cargo Launch Vehicle), the end position is comparatively more important, which the existing vacuum guidance methods can ensure. But, for the missions involving global payload delivery, the desired accuracy is far stringent than that presently expected. For a global payload delivery mission, the flight would comprise of endo, exo, re-entry, and terminal phases of flight which are starkly different from each other. In a typical mission, the first phase is ascending through the atmosphere, controlled by endo-atmospheric guidance system which is generally an open loop method following a preselected profile. Once the altitude is enough to neglect atmospheric effects, the vacuum guidance system takes effect. Here, the transition is made because vacuum guidance methods cannot be applied inside the atmosphere, and the existing endo-atmospheric methods do not offer adequate autonomy so as to ensure the required accuracy. In this paper, we demonstrate the feasibility of a recently proposed pseudospectral model predictive method for exo-atmospheric guidance. This method does not rely on the simplifications of vacuum flight, and yet, it is able to provide on board solution feasible for future application. The method has previously been demonstrated for endo-atmospheric flight, and therefore, it could be a candidate method for the guidance of all phases of global delivery system.

\section{Review of Exo-atmospheric Guidance Methods}

A number of vacuum guidance approaches are available in the existing literature. However, only a few have ever
This is an Open Access article distributed under the terms of the Creative Commons Attribution Non-Commercial License (http://creativecommons.org/licenses/by$\mathrm{nc} / 3.0 /$ ) which permits unrestricted non-commercial use, distribution, and reproduction in any medium, provided the original work is properly cited. (c)

\footnotetext{
* Doctoral Candidate

** Lecturer, Corresponding author: zhouhao.buaa@hotmail.com *** Doctoral Candidate **** Professor
} 
been tested or flown on an actual launch vehicle. The only closed-loop vacuum guidance algorithms applied thus far are as follows: Iterative Guidance Mode (IGM) and Powered Explicit Guidance (PEG). The earliest of these is the Iterative Guidance Mode [1] which was designed for the Saturn class vehicles. The approach was to solve a set of quasi-explicit equations in the near-closed form. However, in this method, the terminal condition was limited to a set, where both the final in-plane velocity components as well as the radial component of the final position coordinates were specified. Compared to IGM, a more general method was developed by G. W. Cherry [2]. His method provided a universal solution to boundaryvalue problems of powered guidance, and derivation of the steering law avoided specialized mathematics. An essential feature of the approach was the E-matrix, which mapped the difference in the boundary conditions into the thrustallocation guidance coefficients. This method was designed to control the final position as well as final components of velocity. It was used for lunar descent and ascent mission of Apollo [3]. A thorough detail of his work can be found in G.W. Cherry's paper [2]. From IGM, a more capable vector based algorithm called Linear Tangent Guidance (LTG) was developed. It was developed by Ronald F. Jaggers. This method was generalized for usage in the Space Shuttle [4] so that it could handle all exo-atmospheric manoeuvres and requirements, including ascent, ascent aborts, and deorbit. LTG was later renamed as PEG and was chosen for Orion orbit insertion, deorbit, and rendezvous burn guidance, and it was also used in the trade studies for Altair lunar landing guidance. The original theoretical formulation of PEG is given in Jaggers [3]. The basic algorithm derives a closed solution to an optimal control problem by maximizing vehicle's total mass at terminus. PEG is formulated under the four important assumptions as follows: the aerodynamic forces are negligible; the engine exhaust velocity is constant; either the thrust (mass flow rate) or acceleration is constant; and the target conditions are independent of time and functions, but only of the estimated (navigation) inertial position and velocity states. With the linear gravity model proposed by Jezewski [5], a costate solution is developed. From optimal control theory and the linear gravity model, it is found that the optimal thrust direction, which is required to steer the vehicle to the desired target, is along the direction of the velocity costate vector. The basic PEG algorithm is designed for handling 4 specific orbital insertion constraints of the final radius, velocity, flight path angle, and orbital inclination. For other forms of terminal constraints, modifications are needed on a case-by-case basis. If the number of terminal constraints is not four, substantial changes to the algorithm will be required. As such, research efforts have continued in expanding the capability of vacuum powered guidance algorithm.

The recent on-going efforts to achieve the capabilities of responsive space launch and autonomous space operations have brought renewed interest in both endo and exo-atmospheric guidance. For guidance inside the atmosphere, numerous methods have been developed and studied. Further details can found in the references [6-10]. However, for exo-atmospheric guidance, the PEG approach still remains as the basis for modification and improvement. Some of the researches on PEG include those of Rose M. Benjamin and David K. Gellar [11], Dukeman [12, 13], Lu, P. et al. [7, 14, 15], Lijun Zhang [16], etc. The methods of Lu, P. et al. $[7,14,15]$ consider complete ascent profile including coast phases. However, the methods involve analytical multiple shooting method for guidance solution and require iterations. Dukeman $[12,13]$ solved calculusof-variation two-point-boundary-value- problem starting from vertical rise till main engine cut-off. The proposed method does not involve iteration and is mathematically faster and robust. Here, we suggest pseudospectral model predictive control for exo as well as endo atmospheric guidance. It is demonstrated that the proposed method can guide a vehicle orbital insertion in a rapid manner.

\section{Pseudospectral Model Predictive Control}

The pseudospectral model predictive control (PMPC) method, which was first presented by Yang Liang et al. [17], combines MPC (Model Predictive Control) and GPM (Gauss Pseudospectral Method). In this approach, the state and control variables are approximated by using LG (Legendre Gauss) points, and the system dynamics are approximated on the LG collocation points. The linear optimal control problem is modelled to obtain corrections to the nominal control, which will minimize terminal deviation from the nominal state. These corrections are derived from the predicted state of the MPC problem. Mathematical formulation of the method is described in the following, with an introduction to GPM.

\subsection{The Gauss Pseudospectral Method}

GPM is a pseudospectral collocation method where a function is interpolated on LG points. For details on GPM, the readers are referred to references $[18,19]$. In this method, the Legendre polynomial $L_{N}(\tau)$ is used as the basis function. 
A Legendre polynomial $L_{N}(\tau)$ of $N$ degree has $N$ LG points which exist in the domain $[-1,1]$. For real problem, the LG points need to be mapped onto real time domain $\left[t_{0}, t_{f}\right]$ using the following affine transformation.

$$
t=\frac{\left(t_{f}-t_{0}\right) \tau+\left(t_{f}+t_{0}\right)}{2}
$$

From pseudospectral law, the derivative of any state collocated on the LG nodes can be approximated as (similar approximation can be used for control profile as well)

$$
\dot{\boldsymbol{x}}^{N}\left(\tau_{k}\right) \approx \frac{2}{\tau_{f}-\tau_{0}}\left({ }^{L G} \bar{D}_{k} \boldsymbol{x}_{l=0}+\sum_{l=1}^{N}{ }^{L G} D_{k l} \boldsymbol{x}_{l}\right)
$$

where the differentiation matrices $D \in \mathbb{R}^{N \times N}$ and $\bar{D} \in \mathbb{R}^{\mathrm{N}}$ are found using the exact derivative of Lagrange interpolating polynomials [18]. These are related to each other by

$$
{ }^{L G} \bar{D}_{k}=-\sum_{l=1}^{N}{ }^{L G} D_{k l}
$$

Gauss pseudospectral approximation to a state's derivative can also be made as

$$
\dot{\boldsymbol{x}}^{N}\left(\tau_{k}\right) \approx \frac{2}{\tau_{f}-\tau_{0}}\left(\sum_{l=1}^{N}{ }^{L G} D_{k l}^{\dagger} \boldsymbol{x}_{l}+{ }^{L G} \bar{D}_{k}^{\dagger} \boldsymbol{x}_{l=N+1}\right)
$$

where $D^{+}$is the adjoint of differential approximation matrix $D$, and their relation [18] is

$$
D_{k l}^{\dagger}=-\frac{\omega_{l}}{\omega_{k}} \cdot D_{l k}
$$

From (2) and (4), we see that the state dynamics can be defined using differential approximation matrix $D$ which is defined for any number of collocation points. As such, in GPM, a system dynamics can be transformed into a set of algebraic equations, which is the core concept of this method.

\subsection{Gauss Pseudospectral Solution to PMPC}

PMPC is a predictive control method where an algebraic solution is formulated by applying GPM. In this method, we assume a nominal trajectory (with state $x^{*}$ and control $u^{*}$ ) and then predict the perturbed trajectory $\left(x^{\mathrm{p}}=x^{*}+\delta x\right)$ by applying the nominal control $u^{*}$ to the perturbed system $x^{p}$. The linear time variant perturbation model is then defined as follows.

$$
\delta \dot{\boldsymbol{x}}=A(t) \delta \boldsymbol{x}+B(t) \delta \boldsymbol{u}
$$

Where the Jacobean matrices are defined as follows (note the superscript ' $p$ ').

$$
A(t)=\frac{\partial f\left(x^{p}(t), u^{p}(t)\right)}{\partial x}, B(t)=\frac{\partial f\left(x^{p}(t), u^{p}(t)\right)}{\partial u}, A \in R^{s \times s}, B \in R^{c \times c}
$$

The optimal control problem can be formed to determine the control deviation $\delta u\left(x^{p}, t\right)$, such that equation (6) is satisfied while minimizing the functional cost

$$
A(t)=\frac{\partial f\left(x^{p}(t), u^{p}(t)\right)}{\partial x}, B(t)=\frac{\partial f\left(x^{p}(t), u^{p}(t)\right)}{\partial u}, A \in R^{s \times s}, B \in R^{c \times c}
$$

The Hamiltonian of the system can be written as

$J=\frac{1}{2} \delta x^{T}\left(t_{f}\right) P_{f} \delta x\left(t_{f}\right)+\frac{1}{2} \int_{t_{f}}^{t_{0}}\left[\left(x^{p}-\delta x\right)^{T} Q\left(x^{p}-\delta x\right)+\left(u^{p}-\delta u\right)^{T}(t) R\left(u^{p}-\delta u\right)\right] d t$

The optimal control is then

$$
\left(\frac{\partial H}{\partial \delta \boldsymbol{u}}\right)^{*}=0 \rightarrow \delta u^{*}(t)=u^{p}-R^{-1}(t) B^{T}(t) \delta \lambda^{*}(t)
$$

The state and costate equations can be obtained as

$$
\begin{aligned}
& \delta \dot{\boldsymbol{x}}^{*}(t)=+\left(\frac{\partial H}{\partial \delta \lambda}\right) * \rightarrow \delta \dot{\boldsymbol{x}}^{*}(t)=A(t) \delta \boldsymbol{x}^{*}(t)+B(t) \delta \boldsymbol{u}^{*}(t) \\
& \delta \dot{\lambda}^{*}(t)=-\left(\frac{\partial H}{\partial \delta x}\right) * \rightarrow \delta \dot{\lambda}^{*}(t)=-Q(t) \delta \boldsymbol{x}^{*}(t)-A^{T}(t) \delta \lambda^{*}(t)+Q x^{p}
\end{aligned}
$$

Substituting the control relation in the state equation, the TPBVP can be stated as

$$
\begin{aligned}
& {\left[\begin{array}{l}
\delta \dot{\boldsymbol{x}}^{*}(t) \\
\delta \dot{\boldsymbol{\lambda}}^{*}(t)
\end{array}\right]=\left[\begin{array}{cc}
\mathrm{A}(t) & -B(t) R^{-1}(t) B^{T}(t) \\
-Q & -A^{T}(t)
\end{array}\right]\left[\begin{array}{l}
\delta \boldsymbol{x}^{*}(t) \\
\delta \boldsymbol{\lambda}^{*}(t)
\end{array}\right]+\left[\begin{array}{l}
B u^{p}(t) \\
Q x^{p}(t)
\end{array}\right]} \\
& \delta x\left(t_{0}\right)=0 \\
& \delta \boldsymbol{\lambda}_{f}=P_{f} \delta \boldsymbol{x}_{f}+v^{T}\left(\frac{\partial \Psi}{\partial \boldsymbol{x}_{f}}\right)
\end{aligned}
$$

Here, equation (14) is the transversatility condition which includes the effect of the predicted state deviation $\Psi$ on the terminal states $x_{f}[17] ; v$ is a Lagrange multiplier. If the desired terminal state is restricted to some specific value, the first part of the transversatility condition is used.

In order to solve this TPBVP, we need to define the derivatives of state and costate on the LG collocation points. This is done as shown in equation (2) and (4). For convenience, we redefine the following $N \times N+1$ matrices

$$
\begin{aligned}
& D=\left[[\bar{D}]_{N \times 1} \quad[D]_{N \times N}\right] \\
& D^{\dagger}=\left[\left[\bar{D}^{\dagger}\right]_{N \times N} \quad\left[\bar{D}^{\dagger}\right]_{N \times 1}\right]
\end{aligned}
$$

The TPBVP can then be transformed into the following set of algebraic equations. These include additional constraints to keep the boundary state and costate variables under the constraints of dynamic equation via Gauss quadrature rule. 


$$
\begin{aligned}
& \sum_{l=0}^{N} D_{k l} \delta x\left(t_{k}\right)=\frac{t_{f}-t_{0}}{2}\left(A_{k} \delta x\left(t_{k}\right)-B_{k} R^{-1} B_{k}^{T} \delta \lambda\left(t_{k}\right)+B_{k} u^{p}\left(t_{k}\right)\right) \\
& \sum_{l=0}^{N} D_{k l} \delta \lambda\left(t_{k}\right)=-\frac{t_{f}-t_{0}}{2}\left(Q \delta x\left(t_{k}\right)+A_{k}^{T} \delta \lambda\left(t_{k}\right)-Q_{k} x^{p}\left(t_{k}\right)\right) \\
& \delta x_{N+1}=\delta x_{0}+\frac{t_{f}-t_{0}}{2} \sum_{k=1}^{N} \omega_{k}\left(A k \delta x\left(t_{k}\right)-B_{k} R^{-1} B_{k}^{T} \delta \lambda\left(t_{k}\right)+B_{k} u^{p}\left(t_{k}\right)\right) \\
& \delta \lambda_{0}=\delta \lambda_{N+1}+\frac{t_{f}-t_{0}}{2} \sum_{k=1}^{N} \omega_{k}\left(Q \delta x\left(t_{k}\right)+A_{k}^{T} \delta \lambda\left(t_{k}\right)-Q_{k} x^{p}\left(t_{k}\right)\right)
\end{aligned}
$$

These algebraic equations can be expressed in the matrix form as

$$
S \Delta z=K
$$

Where, $\Delta \mathrm{z}$ is the column vector consisting of the state and costate deviations, $\delta x$ and $\delta \lambda$. The elements of $S$ and $K$ matrices are defined as

$$
\begin{aligned}
& S=\left[\begin{array}{ll}
S^{x x} & S^{x \lambda} \\
S^{\lambda x} & S^{\lambda \lambda}
\end{array}\right] ; \quad K=\left[\begin{array}{l}
K^{x} \\
K^{\lambda}
\end{array}\right] \\
& S^{x x}=\left[\begin{array}{ccccc}
D_{11}-\frac{t_{f}-t_{0}}{2} A_{1} & D_{12} & \ldots & D_{1 N} & 0 \\
D_{21} & D_{22}-\frac{t_{f}-t_{0}}{2} A_{2} & \ldots & D_{2 N} & 0 \\
\vdots & \vdots & \ddots & \vdots & 0 \\
D_{N 1} & D_{N 2} & \ldots & D_{N N}-\frac{t_{f}-t_{0}}{2} A_{N} & 0 \\
-\omega_{1} \frac{t_{f}-t_{0}}{2} A_{1} & -\omega_{2} \frac{t_{f}-t_{0}}{2} A_{2} & \ldots & -\omega_{N} \frac{t_{f}-t_{0}}{2} A_{N} & 1
\end{array}\right]_{(N+1) \times(N+1)} \\
& S^{x x}=\left[\begin{array}{cccccc}
0 & \frac{t_{f}-t_{0}}{2} B_{1} R^{-1} B_{1}^{T} & 0 & \ldots & 0 & 0 \\
0 & 0 & \frac{t_{f}-t_{0}}{2} B_{2} R^{-1} B_{2}^{T} & \ldots & 0 & 0 \\
0 & \vdots & \vdots & \ddots & \vdots & 0 \\
0 & 0 & 0 & \ldots & \frac{t_{f}-t_{0}}{2} B_{N} R^{-1} B_{N}^{T} & 0 \\
0 & \omega_{1} \frac{t_{f}-t_{0}}{2} B_{1} R^{-1} B_{1}^{T} & \omega_{2} \frac{t_{f}-t_{0}}{2} B_{2} R^{-1} B_{2}^{T} & \ldots & \omega_{N} \frac{t_{f}-t_{0}}{2} B_{N} R^{-1} B_{N}^{T} & 0
\end{array}\right]_{(N+1) \times(N+2)} \\
& S^{\lambda x}=\left[\begin{array}{ccccc}
-\omega_{1} \frac{t_{f}-t_{0}}{2} Q & -\omega_{2} \frac{t_{f}-t_{0}}{2} Q & \cdots & -\omega_{N} \frac{t_{f}-t_{0}}{2} Q & 0 \\
\frac{t_{f}-t_{0}}{2} Q & 0 & \cdots & 0 & 0 \\
0 & \frac{t_{f}-t_{0}}{2} Q & \cdots & 0 & 0 \\
\vdots & \vdots & \ddots & \vdots & \vdots \\
0 & 0 & \cdots & \frac{t_{f}-t_{0}}{2} Q & 0 \\
0 & 0 & \cdots & 0 & P_{f}
\end{array}\right]_{(N+2) \times(N+1)} \\
& S_{\lambda \lambda}=\left[\begin{array}{cccccc}
I & -\omega_{1} \frac{t_{f}-t_{0}}{2} A_{1}^{T} & -\omega_{2} \frac{t_{f}-t_{0}}{2} A_{2}^{T} & \cdots & -\omega_{N} \frac{t_{f}-t_{0}}{2} A_{N}^{T} & -I \\
0 & D_{11}^{\dagger}+\frac{t_{f}-t_{0}}{2} A_{1}^{T} & D_{12}^{\dagger} & \cdots & D_{1 N}^{\dagger} & D_{1(N+1)}^{\dagger} \\
0 & D_{21}^{\dagger} & D_{22}^{\dagger}+\frac{t_{f}-t_{0}}{2} A_{2}^{T} & \cdots & D_{2 N}^{\dagger} & D_{2(N+1)}^{\dagger} \\
0 & \vdots & \vdots & \ddots & \vdots & \vdots \\
0 & D_{N 1}^{\dagger} & D_{N 2}^{\dagger} & \cdots & D_{N N}^{\dagger}+\frac{t_{f}-t_{0}}{2} A_{N}^{T} & D_{N(N+1)}^{\dagger} \\
0 & 0 & 0 & 0 & 0 & -I
\end{array}\right]_{(N+2) \times(N+2)}
\end{aligned}
$$


4. From the new control profile $u_{\mathrm{k}}$ the current control command $u_{0}$ is passed on to the vehicle control system.

5.. The control profile $u_{\mathrm{k}}$ is used in the predictive integration, in order to check the tolerance of projected terminal error.

6.. If the projected terminal error is within limits, this profile replaces the nominal profile and supplies subsequent control commands.

In the above calculation for control profile, equation (17) is solved for which the complete code is written and executed in Matlab. The selected number of collocation node was 10 in this collocation type method. Typical collocation methods are time intensive and generally require higher number of nodes for accuracy. However, the proposed method is a predictive method; as such, it is computationally faster and require lesser number of collocation points. The implementation process is shown in Fig. 1.

\section{Dynamics of Vacuum Flight}

Exo-atmospheric flight ensues after the boundary of dense atmosphere where the guidance system works in a vacuum surrounding. In the exo-atmosphere, the density is significantly low, as to make any aerodynamic effects negligible. Fig. 2 shows the diminishing trend of atmospheric density and air pressure with increasing altitude. In the figure, the dynamic pressure encountered by a point mass vehicle at speeds ranging from 5,000 to $10,000 \mathrm{~m} / \mathrm{sec}$ is also shown. The plots show negligible dynamic pressure above $100 \mathrm{~km}$ altitude where the pressure is around $27 \sim 28 \mathrm{~kg} /\left(\mathrm{m} . \mathrm{sec}^{2}\right)$ for the specified velocity ranges.

Considering the atmospheric condition, vacuum flight can be modelled by completely omitting the aerodynamic effects. The flight mechanical equations can, therefore, be stated as:

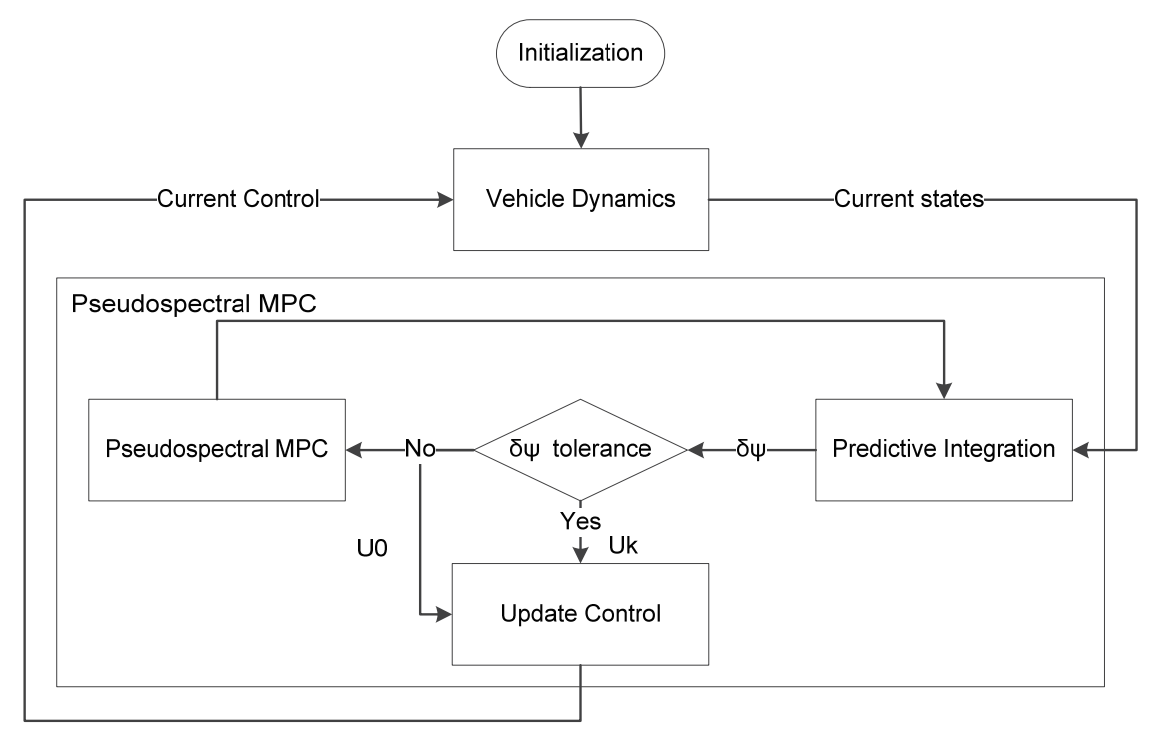

Fig. 1. Implementation steps of Pseudospectral MPC.

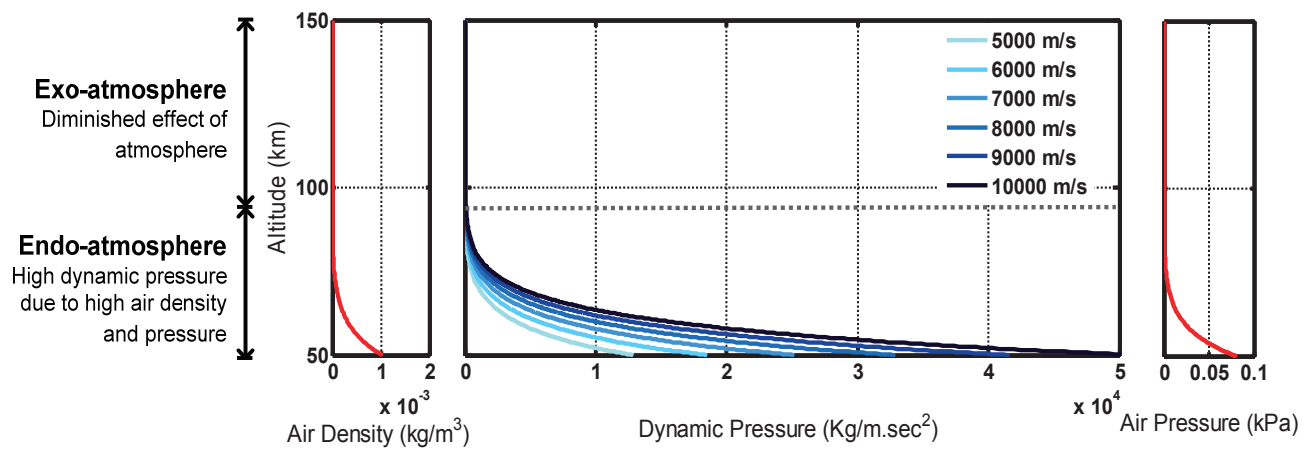

Fig. 2. Reduction of atmospheric effect in the exo-atmosphere. 


$$
\begin{aligned}
& \dot{v}=\frac{T \cos \alpha \cos \sigma}{m}-g \sin \gamma \\
& \dot{\gamma}=\frac{T \sin \alpha}{m v}-\frac{g}{v} \cos \gamma+\frac{v}{r} \cos \gamma \\
& \dot{\chi}=\frac{T \cos \alpha \sin \sigma}{m v^{2} \cos \gamma}-\frac{v}{r} \cos \gamma \cos \chi \tan \phi
\end{aligned}
$$

In the above equations, the effect of Earth's rotation is omitted. Fig. 3 shows the forces acting on a vehicle outside the atmosphere, which are thrust and gravitation. The conventions used for angle of attack $\alpha$ and pitch angle $\theta_{p}$ are also shown in the figure.

Because of the absence of atmosphere, flight in exoatmosphere is considered to have no path constraints, and only physical constraint of control parameter is considered. Moreover, the flight is constrained by end constraints which are represented as:

$$
\begin{array}{lll}
r_{f}-r^{d} \approx 0, & \theta_{f}-\theta^{d} \approx 0, & \varphi_{f}-\varphi^{d} \approx 0 \\
\gamma_{f}-\gamma^{d} \approx 0, & v_{f}-v^{d} \approx 0, & \chi_{f}-\chi^{d} \approx 0
\end{array}
$$

\section{Vehicle Models}

Guidance method presented here is for a hypersonic vehicle in prompt global delivery mission. The complete mission scenario involves launching of the hypersonic vehicle

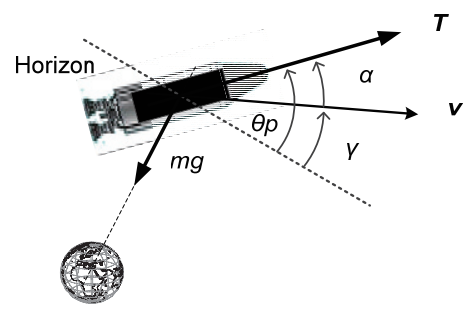

Fig. 3. Forces acting on a flight vehicle in the exo-atmosphere (Longitudinal only).

\section{Launch Vehicle Model \\ Titan II GLV}

$\begin{array}{rl}\text { Payload Mass: } 3580 \mathrm{~kg} & \text { Payload } \\ \text { Total Mass: } 28,587.4 \mathrm{~kg} & \\ \text { Fuel Mass: } 25,839 \mathrm{~kg} & 2 \\ \text { Structural Mass: } 2,748.4 \mathrm{~kg} & \text { Thrust: } 445 \mathrm{kN} \\ & \text { Specific Impulse } 316 \mathrm{sec} \\ & \text { Burn time: } 180 \mathrm{sec} \\ \text { Total Mass: } 122,032.6 \mathrm{~kg} & \text { Stage } 1 \\ \text { Fuel Mass: } 1,17,910 \mathrm{~kg} & \text { Engine: } 1 \times \text { LR-87 } \\ \text { Structural Mass: } 4,122.6 \mathrm{~kg} & \text { Thrust: } 1913 \mathrm{kN} \\ & \text { Specific Impulse } 258 \mathrm{sec} \\ \text { Burn time: } 156 \mathrm{sec}\end{array}$

Fig. 4. Launch vehicle model Titan II GLV.
Table 1. Aerodynamic properties - coefficient of lift for Titan II [21].

\begin{tabular}{|c|c|}
\hline Range of Mach number & Values of $\mathbf{C}_{\mathbf{L} \boldsymbol{\alpha}}$ \\
\hline $0 \leq M a \leq 0.25$ & 2.8 \\
\hline $0.25 \leq M a \leq 1.1$ & $2.8+0.447^{\times}(M a-0.25)$ \\
\hline $1.1 \leq M a \leq 1.6$ & $3.18-0.660^{\times}(M a-1.1)$ \\
\hline $1.6 \leq M a \leq 3.6$ & $2.85+0.350^{\times}(M a-1.6)$ \\
\hline$M a \geq 3.6$ & 3.55 \\
\hline
\end{tabular}

Table 2. Aerodynamic properties - coefficient of drag for Titan II [21].

\begin{tabular}{|c|c|}
\hline Range of Mach number & Values of $\mathbf{C}_{\mathbf{D a}}$ \\
\hline $0 \leq M a \leq 0.8$ & 0.29 \\
\hline $0.8 \leq M a \leq 1.068$ & $M a-0.51$ \\
\hline$M a \geq 1.068$ & $0.091+0.5 \mathrm{Ma}^{-1}$ \\
\hline
\end{tabular}

atop a launch vehicle, release from launch vehicle, re-entry, and finally, reaching the terminus. We assume that Titan II Gemini (GLV) is used for launch. This is a liquid propelled vehicle with a total mass of $154,200 \mathrm{~kg}$, which carries a payload of maximum 3,580 kg [20, 21]. Detailed mass breakdown and propulsion properties are shown in Fig. 4 . The aerodynamic properties are tabulated in Table 1 and 2 [21].

Simplified aerodynamic model of the hypersonic vehicle is represented through the following relations.

$$
\begin{aligned}
& C_{L}=C_{L 0}+C_{L 1} \alpha \\
& C_{D}=C_{D 0}+k C_{L}^{2}
\end{aligned}
$$

Where, $C_{L 0}=-0.01, C_{L 1}=0.018, C_{D 0}=0.01$, and $k=3.4$.

\section{Exo Atmospheric Ascent Guidance}

The flight profile considered here requires strict positioning of the vehicle at each phase of flight. Therefore,

Table 3. Boundary conditions for exo-atmospheric flight.

\begin{tabular}{|c|c|c|}
\hline Parameter & Initial condition & Terminal condition \\
\hline Altitude & $118.64 \mathrm{~km}$ & $144 \mathrm{~km}$ \\
\hline Longitude & $-0.00043^{\circ}$ & $1.9858^{\circ}$ \\
\hline Latitude & $2.044^{\circ}$ & $2.0379^{\circ}$ \\
\hline Velocity & $5418.45 \mathrm{~m} / \mathrm{sec}$ & $6733 \mathrm{~m} / \mathrm{sec}$ \\
\hline Flight path angle & $9.4767^{\circ}$ & $3.3968^{\circ}$ \\
\hline Azimuth angle & $-0.036473^{\circ}$ & $-0.107^{\circ}$ \\
\hline Vehicle mass & $17428.82 \mathrm{~kg}$ & $10900 \mathrm{~kg}$ \\
\hline
\end{tabular}


we need to ensure very accurate end positions which calls for a robust guidance method that can autonomously isolate deviation and steer the vehicle without significant performance degradation. For evaluation, a reference trajectory consisting of two boost and one coast phase is generated using sequential quadratic programming (SQP). For this Matlab command 'fmincon' was used. Fig. 5 below shows the reference profile. The boundary conditions for exo-atmospheric flight are listed in Table 3.

\subsection{Off nominal cases}

Launch vehicle ascent generally employs an open-loop method for endo atmospheric guidance and a closed-loop law for vacuum flight. Here, we consider the launch vehicle using thrust vector for controlling flight. Therefore, the considered perturbations are resulting from problem with the thrust vector control or the engine nozzle.

\subsubsection{Thrust vector control problem}

Thrust vector control (TVC) problems [22-24] may be caused by hardware issues regarding the actuators or power supplies, or it may be due to software issues regarding the improper commands being sent to the actuators. These problems could manifest themselves by actuators failing to hard over (maximum angle), failing in place, or failing to the null position. Typical actuator failures include the followings: 1) lock in place (LIP); 2) hard over failure (HOF); 3) float; and 4) loss of effectiveness (LOE). In the case of LIP failures, the actuator "freezes" at certain condition and does not respond to subsequent commands. HOF is characterized by the actuator moving to and remaining at the upper or lower position limit, regardless of the command. The speed of response is limited by the actuator rate limit. Float failure occurs when the actuator contributes zero moment to the control authority. Loss of effectiveness is characterized by lowering the actuator gain with respect to its nominal value. These actuator problems can be simulated by using the following parameterizations [23].

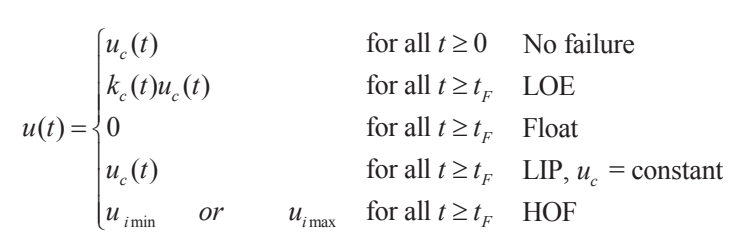

In the above parameterization, $u_{c}$ is the commanded control, $k_{c}$ is the ratio by which the actuator loses effectiveness, and $\mathrm{tF}$ is the moment from when the actuator problem starts. In the case of LIP problem, the $u_{c}(t)$ will remain constant; and for LOE, the commanded control will lose its effectiveness defined by the ratio $k_{c}$. Generally, there are multiple thrust vector nozzles for controlling pitch, yaw and roll motion of launch vehicle. Therefore, simulating the actuator problems require detailed modelling of the thrust vector nozzles. In our evaluation, we only apply loss of control effectiveness.

\subsubsection{Engine nozzle damage}

Engine nozzle damage could occur during flight, resulting in the loss of performance, control issues, or both. This can be represented by the following parameterization of thrust.

$$
T(t)=k_{T} T_{c}(t) \text { for all } \mathrm{t} \geq \mathrm{t}_{\mathrm{F}}
$$

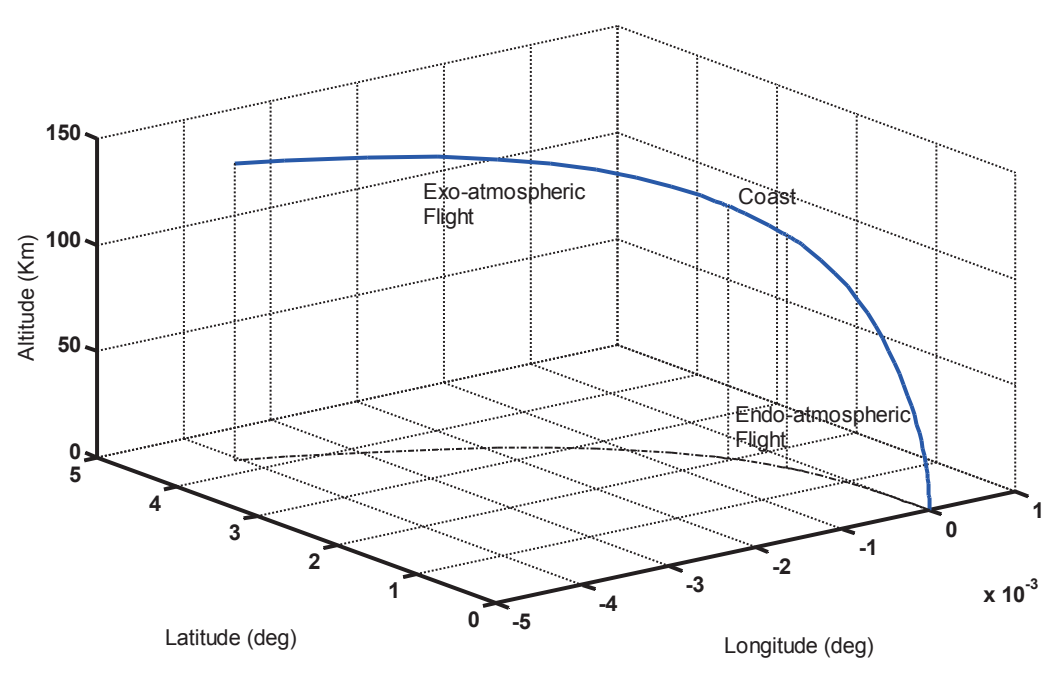

Fig. 5. Ascent trajectory of launch vehicle. 


\subsection{Simulation Results}

Gauss PMPC method for ascent guidance is evaluated through perturbed cases, with thrust and control errors. Guided trajectories for thrust perturbation are compared to unguided trajectories, and the terminal errors are compared. The first set of results is for vector nozzle problem. Here,
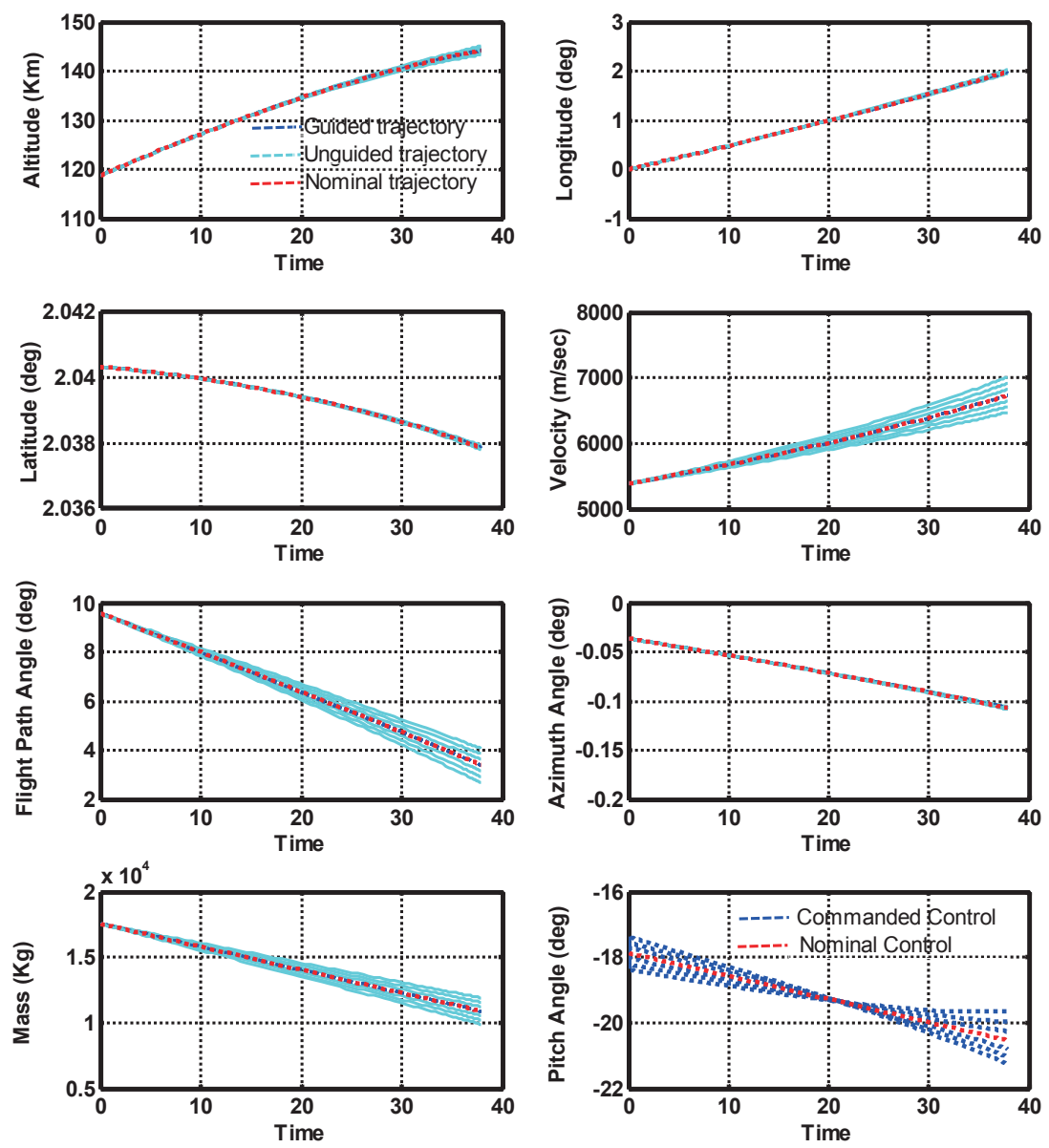

Fig. 6. Guided and unguided trajectory for thrust perturbation (unit of time is seconds).

Table 4. Terminal error for guided and unguided trajectory for thrust perturbation.

\begin{tabular}{|c|c|c|c|c|c|c|c|}
\hline & & $\begin{array}{c}\left|\Delta \mathbf{h}_{\mathrm{f}}\right| \\
(\mathbf{m})\end{array}$ & $\begin{array}{l}\left|\Delta \boldsymbol{\theta}_{\mathrm{f}}\right| \\
(\mathrm{deg})\end{array}$ & $\begin{array}{l}\left|\Delta \varphi_{\mathrm{f}}\right| \\
(\mathrm{deg})\end{array}$ & $\begin{array}{c}\left|\Delta \mathbf{v}_{\mathbf{f}}\right| \\
(\mathbf{m} / \mathbf{s e c})\end{array}$ & $\begin{array}{l}\left|\Delta \gamma_{\mathrm{f}}\right| \\
\text { (deg) }\end{array}$ & $\frac{\left|\Delta \mathbf{m}_{\mathrm{f}}\right|}{(\mathbf{k g})}$ \\
\hline \multirow{6}{*}{ Unguided } & $T=0.85 \times T$ & 862.2 & 0.037 & $6.85 \times 10^{-5}$ & 257.20 & 0.68 & 999.7 \\
\hline & $T=0.90 \times T$ & 584.01 & 0.025 & $4.62 \times 10^{-5}$ & 173.88 & 0.46 & 666.5 \\
\hline & $T=0.95 \times T$ & 296.76 & 0.013 & $2.33 \times 10^{-5}$ & 88.21 & 0.23 & 333.2 \\
\hline & $T=1.05 \times T$ & 306.73 & 0.012 & $2.38 \times 10^{-5}$ & 90.89 & 0.23 & 333.23 \\
\hline & $T=1.10 \times T$ & 623.93 & 0.026 & $4.82 \times 10^{-5}$ & 184.63 & 0.47 & 666.47 \\
\hline & $T=1.15 \times T$ & 952.15 & 0.039 & $7.32 \times 10^{-5}$ & 281.42 & 0.72 & 999.6 \\
\hline \multirow{6}{*}{ Guided } & $T=0.85 \times T$ & 87.4 & $9.08 \times 10^{-5}$ & $1.68 \times 10^{-7}$ & 0.58 & 0.0017 & $<1$ \\
\hline & $T=0.90 \times T$ & 58.86 & $6.17 \times 10^{-5}$ & $1.14 \times 10^{-7}$ & 0.39 & 0.0015 & $<1$ \\
\hline & $T=0.95 \times T$ & 29.72 & $3.15 \times 10^{-5}$ & $5.82 \times 10^{-8}$ & 0.20 & 0.0009 & $<1$ \\
\hline & $T=1.05 \times T$ & 30.26 & $3.28 \times 10^{-5}$ & $6.04 \times 10^{-8}$ & 0.22 & 0.0013 & $<1$ \\
\hline & $T=1.10 \times T$ & 61.04 & $6.65 \times 10^{-5}$ & $1.23 \times 10^{-7}$ & 0.45 & 0.0031 & $<1$ \\
\hline & $T=1.15 \times T$ & 92.3 & $1.02 \times 10^{-5}$ & $1.88 \times 10^{-7}$ & 0.70 & 0.0055 & $<1$ \\
\hline
\end{tabular}


mass is maintained to less than $1 \mathrm{~kg}$.

Control problem has more prominent effect on the altitude and flight path angle. Error in vehicle mass is almost negligible. The results for control perturbation in Fig. 7 and Table 5 show that the method is capable of minimizing the effects. The terminal altitude error was within 100 meters; terminal flight path angle was also within the acceptable
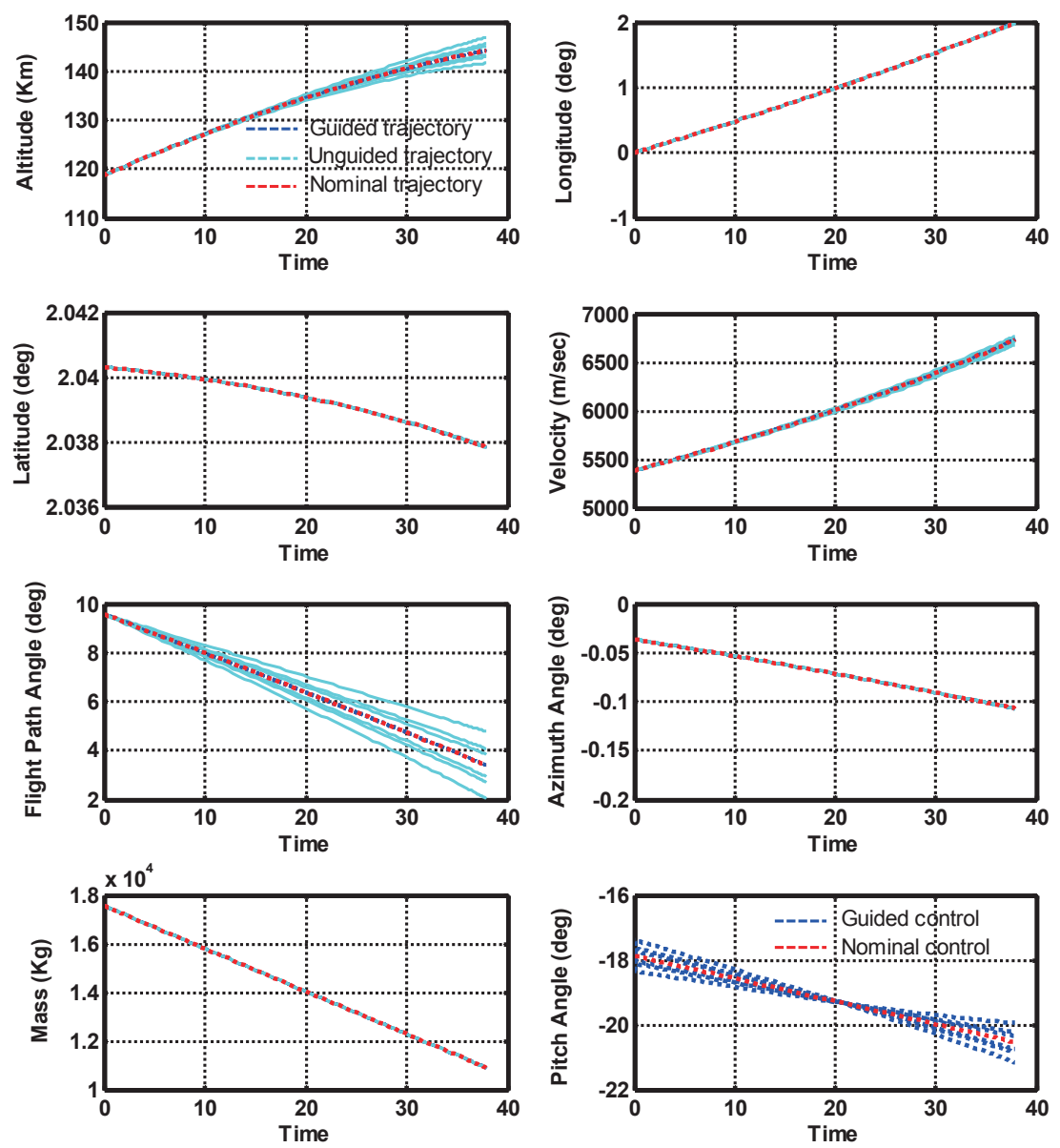

Fig. 7. Guided and unguided trajectory for control perturbations (unit of time is seconds).

Table 5. Terminal error for guided and unguided trajectory for control perturbation.

\begin{tabular}{|c|c|c|c|c|c|c|}
\hline & & $\begin{array}{c}\left|\Delta \mathbf{h}_{\mathrm{f}}\right| \\
(\mathbf{m})\end{array}$ & $\begin{array}{l}\left|\Delta \theta_{\mathrm{f}}\right| \\
(\mathbf{d e g})\end{array}$ & $\begin{array}{l}\left|\Delta \varphi_{\mathrm{f}}\right| \\
(\mathrm{deg})\end{array}$ & $\begin{array}{c}\left|\Delta \mathbf{v}_{\mathrm{f}}\right| \\
(\mathrm{m} / \mathrm{sec})\end{array}$ & $\begin{array}{l}\left|\Delta \gamma_{\mathrm{f}}\right| \\
\text { (deg) }\end{array}$ \\
\hline \multirow{6}{*}{ Unguided } & $k_{c}=0.70$ & 2674.36 & 0.0029 & $5.54 \times 10^{-6}$ & 38.00 & 1.38 \\
\hline & $k_{c}=0.85$ & 1331.93 & 0.0018 & $3.35 \times 10^{-6}$ & 20.82 & 0.69 \\
\hline & $k_{c}=0.90$ & 886.59 & 0.0013 & $2.36 \times 10^{-6}$ & 14.27 & 0.45 \\
\hline & $k_{c}=1.10$ & 880.14 & 0.0016 & $2.87 \times 10^{-6}$ & 15.87 & 0.46 \\
\hline & $k_{c}=1.15$ & 1317.42 & 0.0024 & $4.49 \times 10^{-6}$ & 24.39 & 0.68 \\
\hline & $k_{c}=1.30$ & 2616.39 & 0.0055 & $1.01 \times 10^{-5}$ & 52.29 & 1.35 \\
\hline \multirow{6}{*}{ Guided } & $\mathbf{k}_{\mathrm{c}}=\mathbf{0 . 7 0}$ & 85 & $8.78 \times 10^{-5}$ & $1.62 \times 10^{-7}$ & 0.49 & 0.0004 \\
\hline & $k_{c}=0.85$ & 42.16 & $4.45 \times 10^{-5}$ & $8.22 \times 10^{-8}$ & 0.24 & 0.0001 \\
\hline & $k_{c}=0.90$ & 28.03 & $2.98 \times 10^{-5}$ & $5.5 \times 10^{-8}$ & 0.16 & $5.6 \times 10^{-5}$ \\
\hline & $k_{c}=1.10$ & 27.71 & $3.02 \times 10^{-5}$ & $5.58 \times 10^{-8}$ & 0.15 & $3.1 \times 10^{-5}$ \\
\hline & $k_{c}=1.15$ & 41.43 & $4.54 \times 10^{-5}$ & $8.4 \times 10^{-8}$ & 0.21 & $8.2 \times 10^{-5}$ \\
\hline & $k_{c}=1.30$ & 82.07 & $9.17 \times 10^{-5}$ & $1.69 \times 10^{-7}$ & 0.40 & 0.00038 \\
\hline
\end{tabular}


is therefore imperative to assess the presented method for the degree of autonomy it can offer. The method is by formulation, a predictive one, where a reference control profile is corrected based on the terminal error in the predicted trajectory. However, it is not important
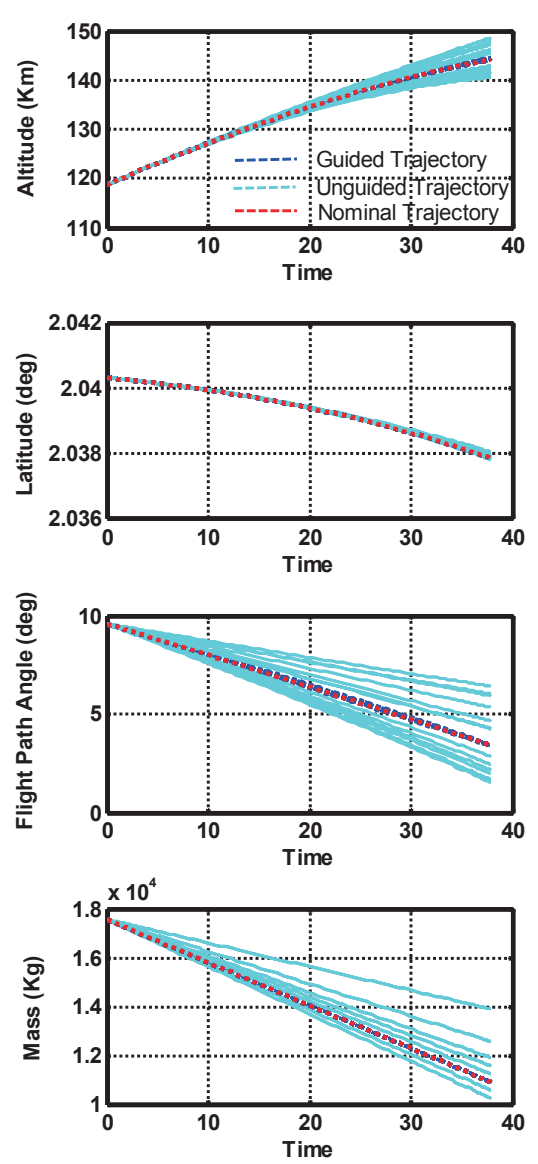

that an optimal control profile is available for the initial predictions. Any arbitrary profile should be adequate, as long as the predicted trajectory is not too far from the required one. In order to ascertain the sensitivity of initial control guess, we apply a 'zero' profile as the initial
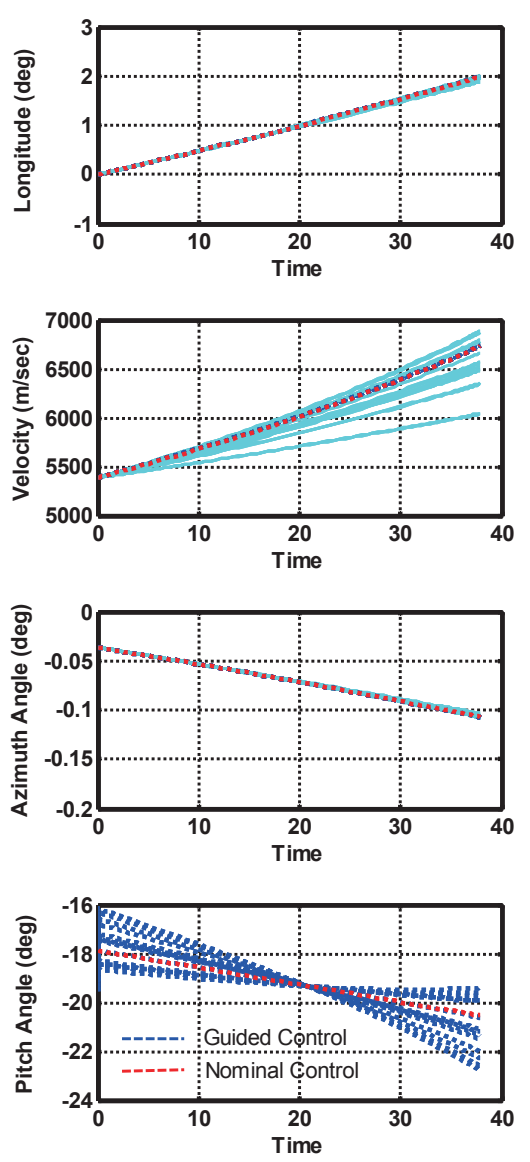

Fig. 8. Guided and unguided trajectories for both control and thrust perturbations (unit of time is seconds).

Table 6. Terminal error for guided trajectory with both control and thrust problems.

\begin{tabular}{|c|c|c|c|c|c|c|c|}
\hline $\mathbf{k c}$ & $\mathbf{T}$ & $\begin{array}{c}\left|\Delta \mathbf{h}_{\mathbf{f}}\right| \\
(\mathbf{m})\end{array}$ & $\begin{array}{c}\left|\Delta \boldsymbol{\theta}_{\mathbf{f}}\right| \\
(\mathbf{d e g})\end{array}$ & $\begin{array}{c}\left|\Delta \boldsymbol{\varphi}_{\mathbf{f}}\right| \\
(\mathbf{d e g})\end{array}$ & $\begin{array}{c}\left|\Delta \mathbf{v}_{\mathbf{f}}\right| \\
(\mathbf{m} / \mathbf{s e c})\end{array}$ & $\begin{array}{c}\left|\Delta \mathbf{\gamma}_{\mathbf{f}}\right| \\
(\mathbf{d e g})\end{array}$ & $\begin{array}{c}\left|\Delta \mathbf{m}_{\mathbf{f}}\right| \\
(\mathbf{k g})\end{array}$ \\
\hline $\mathbf{1 . 1 5}$ & $\mathbf{1 . 0 5}$ & 74.99 & $8.32 \times 10^{-5}$ & $1.54 \times 10^{-7}$ & 0.44 & 0.0012 & $<1$ \\
\hline $\mathbf{1 . 2 5}$ & $\mathbf{1 . 1 0}$ & 140.94 & 0.00016 & $2.97 \times 10^{-7}$ & 0.81 & 0.0027 & $<1$ \\
\hline $\mathbf{1 . 2 5}$ & $\mathbf{1 . 0 5}$ & 104.39 & 0.00012 & $2.17 \times 10^{-7}$ & 0.57 & 0.0009 & $<1$ \\
\hline $\mathbf{1 . 1 5}$ & $\mathbf{1 . 1 0}$ & 109.22 & 0.00012 & $2.26 \times 10^{-7}$ & 0.68 & 0.0029 & $<1$ \\
\hline $\mathbf{1 . 5 0}$ & $\mathbf{0 . 9 5}$ & 94.61 & 0.00016 & $1.96 \times 10^{-7}$ & 0.40 & 0.0017 & $<1$ \\
\hline $\mathbf{1 . 2 5}$ & $\mathbf{0 . 9 0}$ & $<1$ & $8.74 \times 10^{-7}$ & $1.61 \times 10^{-9}$ & 0.07 & 0.0015 & $<1$ \\
\hline $\mathbf{0 . 5 5}$ & $\mathbf{0 . 5 5}$ & 301.57 & 0.00028 & $5.24 \times 10^{-7}$ & 1.97 & 0.0010 & $<1$ \\
\hline $\mathbf{0 . 7 5}$ & $\mathbf{0 . 5 5}$ & 276.41 & 0.00026 & $4.89 \times 10^{-7}$ & 1.76 & 0.0004 & $<1$ \\
\hline $\mathbf{0 . 5 5}$ & $\mathbf{0 . 7 5}$ & 227.38 & 0.00022 & $4.06 \times 10^{-7}$ & 1.52 & 0.0026 & $<1$ \\
\hline $\mathbf{0 . 7 5}$ & $\mathbf{0 . 7 5}$ & 189.11 & 0.00019 & $3.46 \times 10^{-7}$ & 1.24 & 0.0022 & $<1$ \\
\hline $\mathbf{0 . 9 0}$ & $\mathbf{0 . 9 0}$ & 82.77 & $8.56 \times 10^{-5}$ & $1.58 \times 10^{-7}$ & 0.54 & 0.0016 & $<1$ \\
\hline $\mathbf{0 . 8 5}$ & $\mathbf{0 . 8 5}$ & 120.49 & 0.00012 & $2.27 \times 10^{-7}$ & 0.79 & 0.00198 & $<1$ \\
\hline $\mathbf{0 . 9 5}$ & $\mathbf{0 . 9 5}$ & 42.63 & $4.48 \times 10^{-5}$ & $8.29 \times 10^{-8}$ & 0.28 & 0.00095 & $<1$ \\
\hline $\mathbf{0 . 8 5}$ & $\mathbf{0 . 9 5}$ & 68.72 & $7.15 \times 10^{-5}$ & $1.32 \times 10^{-7}$ & 0.43 & 0.0010 & $<1$ \\
\hline $\mathbf{0 . 9 5}$ & $\mathbf{0 . 8 5}$ & 98.37 & 0.00010 & $1.87 \times 10^{-7}$ & 0.65 & 0.0018 & $<1$ \\
\hline
\end{tabular}


Table 7. Terminal error for unguided trajectory with both control and thrust problems.

\begin{tabular}{|c|c|c|c|c|c|c|c|}
\hline $\mathbf{k c}$ & $\mathbf{T}$ & $\begin{array}{c}\left|\Delta \mathbf{h}_{\mathbf{f}}\right| \\
(\mathbf{m})\end{array}$ & $\begin{array}{c}\left|\Delta \mathbf{\theta}_{\mathbf{f}}\right| \\
(\mathbf{d e g})\end{array}$ & $\begin{array}{c}\left|\Delta \boldsymbol{\varphi}_{\mathrm{f}}\right| \\
(\mathbf{d e g})\end{array}$ & $\begin{array}{c}\left|\Delta \mathbf{v}_{\mathbf{f}}\right| \\
(\mathbf{m} / \mathbf{s e c})\end{array}$ & $\begin{array}{c}\left|\Delta \mathbf{y}_{\mathbf{f}}\right| \\
(\mathbf{d e g})\end{array}$ & $\begin{array}{c}\left|\Delta \mathbf{m}_{\mathbf{f}}\right| \\
(\mathbf{k g})\end{array}$ \\
\hline $\mathbf{1 . 1 5}$ & $\mathbf{1 . 0 5}$ & 1707.41 & 0.0102 & $1.89 \times 10^{-7}$ & 64.88 & 0.95 & 333.23 \\
\hline $\mathbf{1 . 2 5}$ & $\mathbf{1 . 1 0}$ & 3089.19 & 0.0208 & $3.88 \times 10^{-7}$ & 136.27 & 1.74 & 667 \\
\hline $\mathbf{1 . 2 5}$ & $\mathbf{1 . 0 5}$ & 2630.38 & 0.0081 & $1.51 \times 10^{-5}$ & 45.45 & 1.43 & 331.2 \\
\hline $\mathbf{1 . 1 5}$ & $\mathbf{1 . 1 0}$ & 2110.08 & 0.0231 & $4.30 \times 10^{-5}$ & 156.94 & 1.23 & 666.46 \\
\hline $\mathbf{1 . 5 0}$ & $\mathbf{0 . 9 5}$ & 3751.74 & 0.0223 & $4.13 \times 10^{-5}$ & 176.96 & 1.87 & 333.23 \\
\hline $\mathbf{1 . 2 5}$ & $\mathbf{0 . 9 0}$ & 1336.02 & 0.0288 & $5.31 \times 10^{-5}$ & 211.10 & 0.54 & 666.47 \\
\hline $\mathbf{0 . 5 5}$ & $\mathbf{0 . 5 5}$ & 4350.21 & 0.1049 & 0.00019 & 688.57 & 3.02 & 2999.09 \\
\hline $\mathbf{0 . 7 5}$ & $\mathbf{0 . 5 5}$ & 3462.76 & 0.1053 & 0.00019 & 697.66 & 2.54 & 2999.09 \\
\hline $\mathbf{0 . 5 5}$ & $\mathbf{0 . 7 5}$ & 4231.41 & 0.0587 & 0.00010 & 381.26 & 2.62 & 1666.16 \\
\hline $\mathbf{0 . 7 5}$ & $\mathbf{0 . 7 5}$ & 2964.08 & 0.0593 & 0.00011 & 394.35 & 1.94 & 1666.16 \\
\hline $\mathbf{0 . 9 0}$ & $\mathbf{0 . 9 0}$ & 1362.47 & 0.0239 & $4.41 \times 10^{-5}$ & 161.42 & 0.86 & 666.46 \\
\hline $\mathbf{0 . 8 5}$ & $\mathbf{0 . 8 5}$ & 1953.38 & 0.0357 & $6.59 \times 10^{-5}$ & 240.26 & 1.25 & 999.70 \\
\hline $\mathbf{0 . 9 5}$ & $\mathbf{0 . 9 5}$ & 711.99 & 0.0119 & $2.22 \times 10^{-5}$ & 81.34 & 0.45 & 333.23 \\
\hline $\mathbf{0 . 8 5}$ & $\mathbf{0 . 9 5}$ & 1546.39 & 0.011 & $2.02 \times 10^{-5}$ & 68.73 & 0.88 & 333.23 \\
\hline $\mathbf{0 . 9 5}$ & $\mathbf{0 . 8 5}$ & 1224.82 & 0.0367 & $6.75 \times 10^{-5}$ & 251.21 & 0.86 & 999.69 \\
\hline
\end{tabular}

control. In Fig. 9, we see that in the 3rd correction, the control profile from Gauss PMPC almost exactly matches the optimal one.

In Fig. 10, the altitude predictions are shown for the above control profiles. From the plot, it is evident that even with a deviation of around $7 \mathrm{~km}$ in initial prediction (with arbitrarily selected control profile), the proposed method could close in near to the optimal solution in the

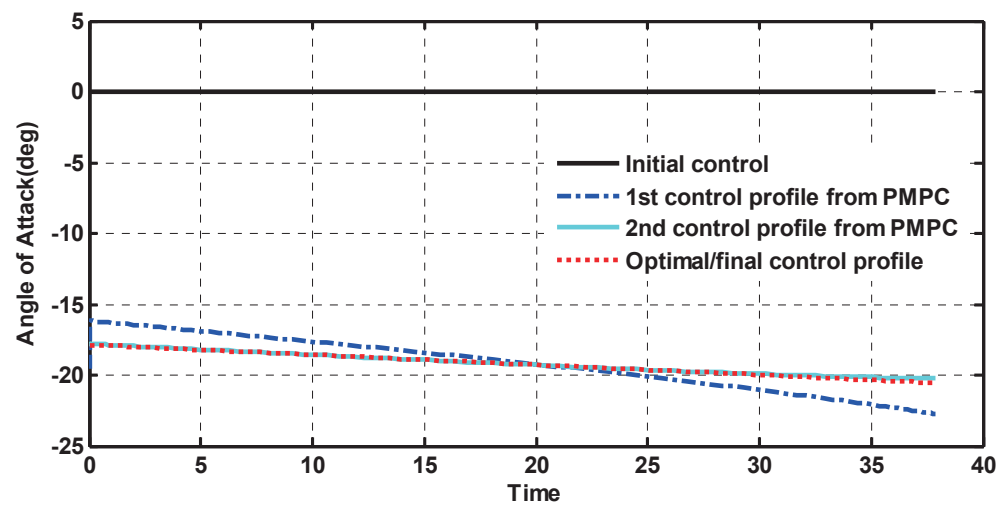

Fig. 9. Initial, corrected, and optimal control profiles (unit of time is seconds).

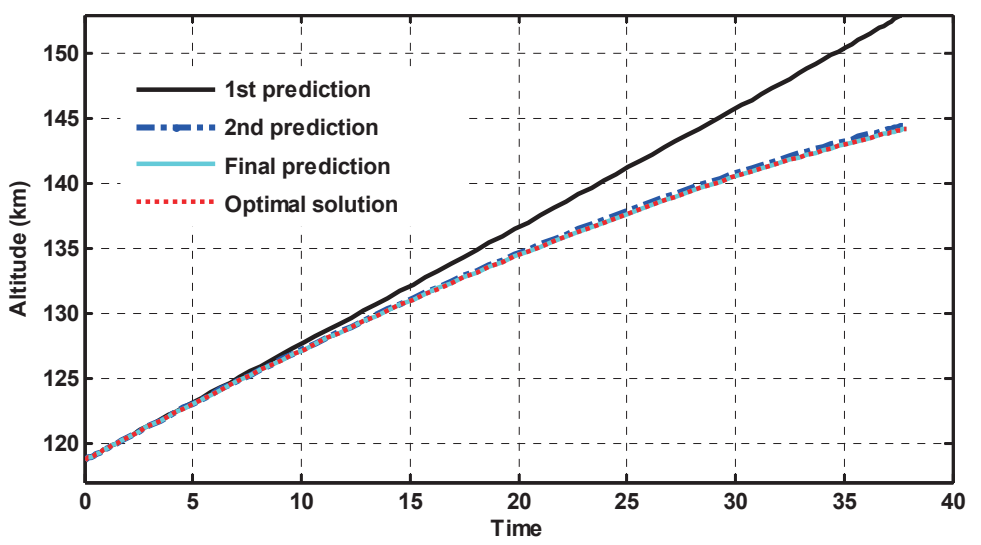

Fig. 10. Predicted and optimal altitude profiles (unit of time is seconds). 
Table 8. Terminal state errors for predicted and optimal control profiles.

\begin{tabular}{|c|c|c|c|c|c|}
\hline Control profile & $\begin{array}{c}\left|\Delta \mathbf{h}_{\mathrm{f}}\right| \\
(\mathbf{m})\end{array}$ & $\begin{array}{c}\left|\Delta \boldsymbol{\theta}_{\mathrm{f}}\right| \\
(\mathbf{d e g})\end{array}$ & $\begin{array}{c}\left|\Delta \boldsymbol{\varphi}_{\mathrm{f}}\right| \\
(\mathbf{d e g})\end{array}$ & $\begin{array}{c}\left|\Delta \mathbf{v}_{\mathrm{f}}\right| \\
(\mathbf{m} / \mathbf{s e c})\end{array}$ & $\begin{array}{c}\left|\Delta \mathbf{y}_{\mathrm{f}}\right| \\
(\mathbf{d e g})\end{array}$ \\
\hline Initial guess & 7992.7365 & 0.0002732 & $-5.0489 \mathrm{e}-007$ & -1.9872 & -0.0027412 \\
\hline $1^{\text {st }}$ control correction & 8.4472 & $1.3 \mathrm{e}-005$ & $-2.4121 \mathrm{e}-008$ & 0.39154 & 0.010032 \\
\hline $2^{\text {nd }}$ control correction & 0.90389 & $9.6461 \mathrm{e}-007$ & $-1.7812 \mathrm{e}-009$ & -0.023269 & -0.00041848 \\
\hline
\end{tabular}

very first step. The optimal solution was reached in the 3rd step with very negligible terminal errors (shown in Table 8).

\section{Conclusion}

A pseudospectral model predictive control method is proposed for exo-atmospheric guidance problem. The method is robust, because it relies on predicted trajectory for generating guidance solution. The method also has significant autonomy, because it does not necessarily need an optimal initial guess. The method is evaluated for thrust and control problems. The results indicate its feasibility in rejecting perturbations. However, the proposed method has only been applied for exo-atmospheric guidance; it does not consider the complete ascent flight. Further work is necessary to consider the complete ascent flight, including coast phases, in order to have a truly effective ascent guidance method.

\section{References}

[1] Smith, I.E., General Formulation of the Iterative Guidance Mode. 1966.

[2] Cherry, G., A general, explicit, optimizing guidance law for rocket-propelled spaceflight. 1964.

[3] Jaggers, R.F., An explicit solution to the exoatmospheric powered flight guidance and trajectory optimization problem for rocket propelled vehicles. 1977.

[4] W, S., The Space Shuttle ascent guidance and control, in Guidance and Control Conference. American Institute of Aeronautics and Astronautics.1982.

[5] Jezewski, D.J., "An optimal, analytic solution to the linear- gravity, constant-thrust trajectory problem", Journal of Spacecraft and Rockets,Vol. 8, No. 7, 1971, pp. 793-796.

[6] Lu, P., et al., "Rapid Optimal Multiburn Ascent Planning and Guidance”, Journal of Guidance, Control, and Dynamics, Vol. 31, No. 6, 2008, pp. 1656-1664.

[7] Ping, L., Z. Lijun. and S. Hongsheng, Ascent Guidance for Responsive Launch: A Fixed-Point Approach, in AIAA
Guidance, Navigation, and Control Conference and Exhibit, American Institute of Aeronautics and Astronautics, 2005.

[8] Lu, P. and J. Samsundar, "Closed form solutions of constrained trajectories application in optimal ascent of aerospace plane", in AIAA International Aerospace Planes Conference, AIAA: Orlando, FL, 1992.

[9] Zhou, H., T. Rahman, and W. Chen, "Neural network assisted inverse dynamic guidance for terminally constrained entry flight", ScientificWorldJournal, 2014. 2014: pp. 686040.

[10] Zhou, H., et al., "Onboard pseudospectral guidance for re-entry vehicle", Proceedings of the Institution of Mechanical Engineers, Part G: Journal of Aerospace Engineering, 2013.

[11] Rose, M.B. and D. Geller, "Linear Covariance Techniques for Powered Ascent", in AIAA Guidance, Navigation, and Control Conference, Americal Institute of Aeronautics and Astronautics: Toronto, Ontario Canada, 2010 .

[12] Greg, D., "Atmospheric Ascent Guidance for RocketPowered Launch Vehicles", in AIAA Guidance, Navigation, and Control Conference and Exhibit, American Institute of Aeronautics and Astronautics, 2002.

[13] Dukeman, G. and A. Calise, "Enhancements to an Atmospheric Ascent Guidance Algorithm", in AIAA Guidance, Navigation, and Control Conference and Exhibit, AIAA: Austin, Texas, 2003.

[14] Lu, P., S. Forbes, and M. Baldwin, "A Versatile Powered Guidance Algorithm", in AIAA Guidance, Navigation, and Control Conference, American Institute of Aeronautics and Astronautics: Minneapolis, Minnesota, 2012.

[15] Lu, P. and B. Pan, "Highly Constrained Optimal Launch Ascent Guidance”, Journal of Guidance, Control, and Dynamics, Vol. 33, No. 2, 2010, pp. 404-414.

[16] Zhang, L. and P. Lu, "Fixed-point algorithms for optimal ascent trajectories of launch vehicles", Engineering Optimization, Vol. 40, No. 4, 2008, pp. 361-381.

[17] Yang, L., H. Zhou, and W. Chen, "Application of linear gauss pseudospectral method in model predictive control", Acta Astronautica, Vol. 96, 2014, pp. 175-187.

[18] Benson, D., A Gauss Pseudospectral Transcription for Optimal Control, Massachusetts Institute of Technology, 
2005.

[19] Garg, D., W. Hager, and A. Rao, Gauss Pseudospectral Method for Solving Infinite-Horizon Optimal Control Problems. 2010.

[20] Andrews, D.G., An Introduction of Atmospheric Physics.

[21] He, L., Solid Ballistic Missiles Design, Beihang University (BUAA)Press, 2004.

[22] Boskovic, J.D., et al., "Multiple-Model Adaptive Fault-Tolerant Control of a Planetary Lander", Journal of
Guidance, Control, and Dynamics, Vol. 32, No. 6, 2009, pp. 1812-1826.

[23] Boskovic, J.D., S. Bergstrom, and R.K. Mehra, "Robust Integrated Flight Control Design Under Failures, Damage, and State-Dependent Disturbances", Journal of Guidance, Control, and Dynamics, Vol. 28, No. 5, 2005, pp. 902-917.

[24] Hanson, J., A.D. Hill, and B.B. Beard, "Launch Vehicle Abort Analysis of Failures Leading to Loss of Control", Journal of Spacecraft and Rockets, Vol. 49, No. 5, 2012, pp. 925-934. 